\title{
Eight powers of ten: similarities in black hole accretion on all mass scales
}

\section{Rob Fender*}

University of Southampton, UK

E-mail: rpf@phys.soton.ac.uk

\section{Elmar Körding}

University of Southampton, UK

\section{Tomaso Belloni}

Osservatorio Astronomico di Brera, Italy

\section{Phil Uttley}

University of Amsterdam, $N L$

\section{lan McHardy}

University of Southampton, UK

\section{Tasso Tzioumis}

ATNF, Australia

In this paper we discuss the recent advances in the quantitative comparison of accretion, and the accretion:jet coupling, in accreting black holes in both X-ray binaries (where $M_{\mathrm{BH}} \sim 10 M_{\odot}$ ) and Active Galactic Nuclei $\left(10^{5} \leq M_{\mathrm{BH}} \leq 10^{9}\right)$. These similarities include the radiative efficiency and jet power as a function of accretion rate, which are themselves probably the origin of the 'fundamental plane of black hole activity'. A second 'fundamental plane' which connects mass, accretion rate and timing properties provides us with a further physical diagnostic. Patterns of radio loudness (i.e. jet production) as a function of luminosity and accretion state are shown to be similar for X-ray binaries and AGN. Finally we discuss how neutron stars are a useful control sample, and what the future may hold for this field.

VI Microquasar Workshop: Microquasars and Beyond

September 18-22 2006

Società del Casino, Como, Italy

\footnotetext{
*Speaker.
} 


\section{Similarities between XRB and AGN: not a new idea...}

Black holes are extremely simple objects which we find at large in our universe over an enormous range (at least eight orders of magnitude) of physical scales. At both extremes of this scale their most obvious property is their copious power output as a result of accretion. In this paper some recent results on the similarity of this accretion process, and the related outflows, across this extreme range of scale, will be discussed. Before we do so it is important however to note that the concept that black hole accretion might in some sense be scale-invariant, or only weakly dependent on scale, is not new.

In 1976, Shakura \& Sunyaev clearly outlined what they expected to be the simple scaling laws between stellar mass black holes in X-ray binary systems, and supermassive black holes in quasars. McHardy (1988) noted similarities in the power spectra of both classes of system, and Sams, Eckart \& Sunyaev (1996) discussed the scaling of black hole jets with mass (a discussion expanded upon in some detail by Heinz \& Sunyaev (2003)). Falcke \& Biermann (1996) further elaborated in some detail on the likely accretion:jet coupling in black holes on all scales, while Pounds, Done \& Osborne (1995) discussed the similarities between Seyfert X-ray emission and that of black hole X-ray binaries in soft X-ray states. Famously, Mirabel et al. (1992) and Mirabel \& Rodriguez $(1994,1999)$ coined the phrase 'microquasar' for jet-producing X-ray binaries, inescapably fixing the analogy in the minds of all researchers. Markoff et al. $(2001,2003)$ have taken models inspired by low-luminosity AGN and adapted them to the hard state of black hole X-ray binaries. Most recently, Maccarone, Fender \& Ho (2005) comprises a compilation of papers on this subject.

\section{Why we expect black hole accretion to be essentially scale-free}

Extreme mathematical simplicity! For all black holes, the ratio between the mass and radius of the event horizon, $M / R$, is the same, to within a factor of

- two if we consider $\mathrm{R}$ at the event horizon

- six if we consider R at the Innermost Stable Circular Orbit (ISCO)

- in both cases the largest R corresponds to a non-spinning (Schwarzschild) black hole, and the smallest $\mathrm{R}$ to a maximally rotating ('Maximal Kerr') black hole.

There are no other parameters (besides charge, which is generally considered to be unimportant), i.e. black holes have no hair (e.g. Carter 1973). Characteristic timescales such as the orbital period at the innermost stable circular orbit likewise scale linearly with mass. Observations indicate an observed range of masses, and therefore of physical scales, of about $10^{8}$ over which this scaling should hold. No other objects in the universe scale so perfectly.

\section{Why we do not expect black hole accretion to be essentially scale-free}

Microphysics! The matter at the inner edge (or elsewhere) of an X-ray binary accretion disc is much hotter and probably much denser than that in an accretion disc around a supermassive black hole (and who knows about magnetic field?). 


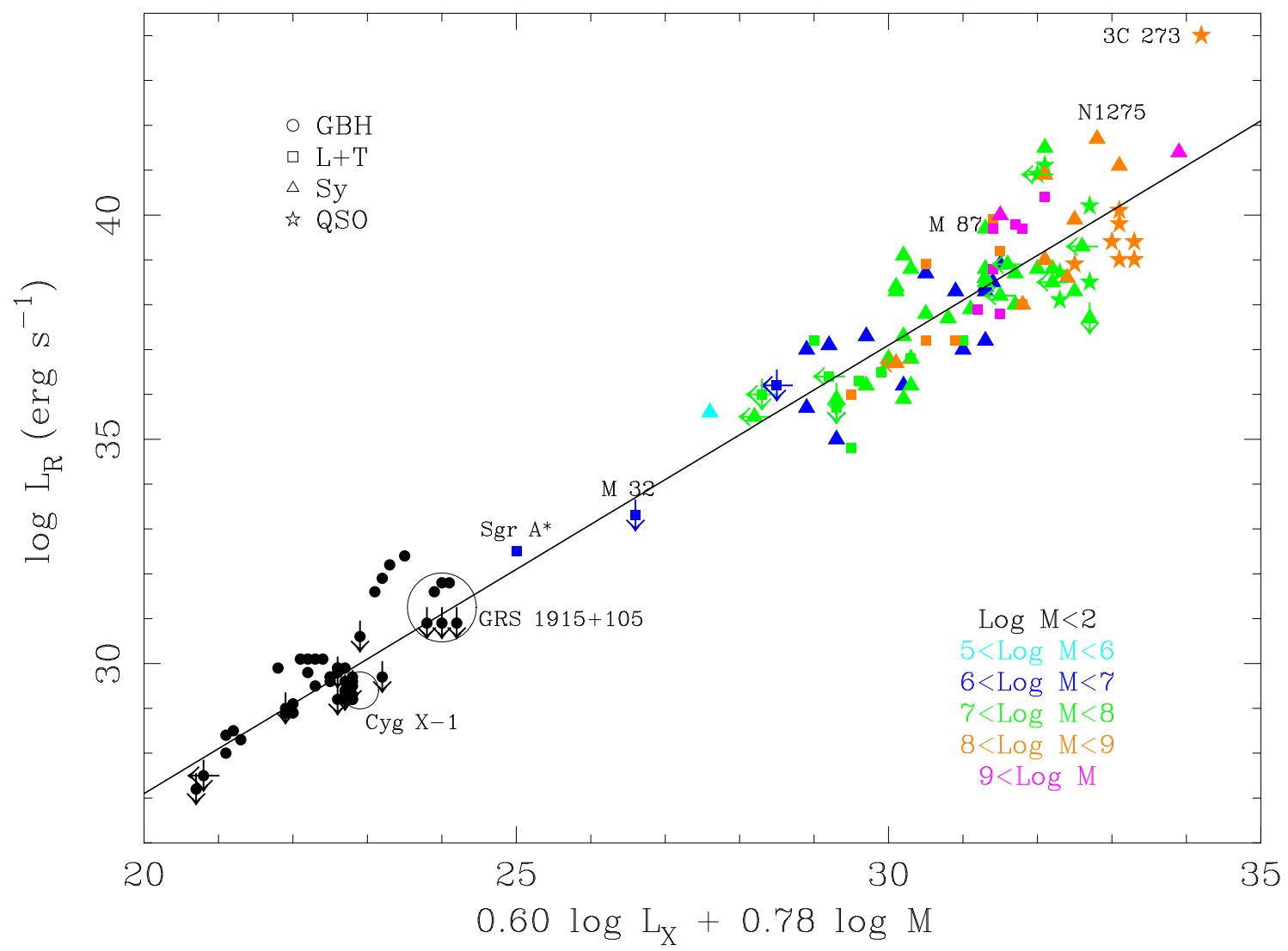

Figure 1: The fundamental plane of black hole activity, relating radio luminosity, X-ray luminosity and mass over more than eight orders of magnitude in black hole mass. A major step forward in the unification of accreting black holes on all mass scales. From Merloni, Heinz \& di Matteo (2003); Falcke, Körding \& Markoff (2004) independently arrived at almost the same result.

\section{The fundamental plane of black hole activity}

Corbel et al. $(2000,2003)$ demonstrated a clear nonlinear relation between the radio luminosty $L_{\text {radio }}$ and X-ray luminosity, $L_{X}$ in the black hole binary GX 339-4 (this source is a fantastic testbed for such studies since it regularly varies by $4-5$ order of magnitude in $L_{X}$ and has no detectable / contaminating companion star), of the form

$$
L_{\text {radio }} \propto L_{X}^{0.7}
$$

Gallo, Fender \& Pooley (2003), compiled a larger sample of more black hole binaries and found this relation to be essentially universal, an assertion recently confirmed by the detection of radio emission from the quiescent $\left(L_{X} \sim 10^{-8} L_{\text {Edd }}\right)$ black hole binary A 0620-00 at the level predicted by the relation (Gallo et al. 2006; note the exponent of the correlation flattens slightly to +0.6 when this quiescent source is included). However, it should be noted that an increasing number of sources are being found at relatively high luminosities $\left(L_{X} \geq 10^{-3} L_{\text {Edd }}\right)$ which drop off the correlation (Gallo 2007). Some possible explanations for this effect could include partial quenching or increased velocity of the jets as the soft state is approached, but more data are required. 


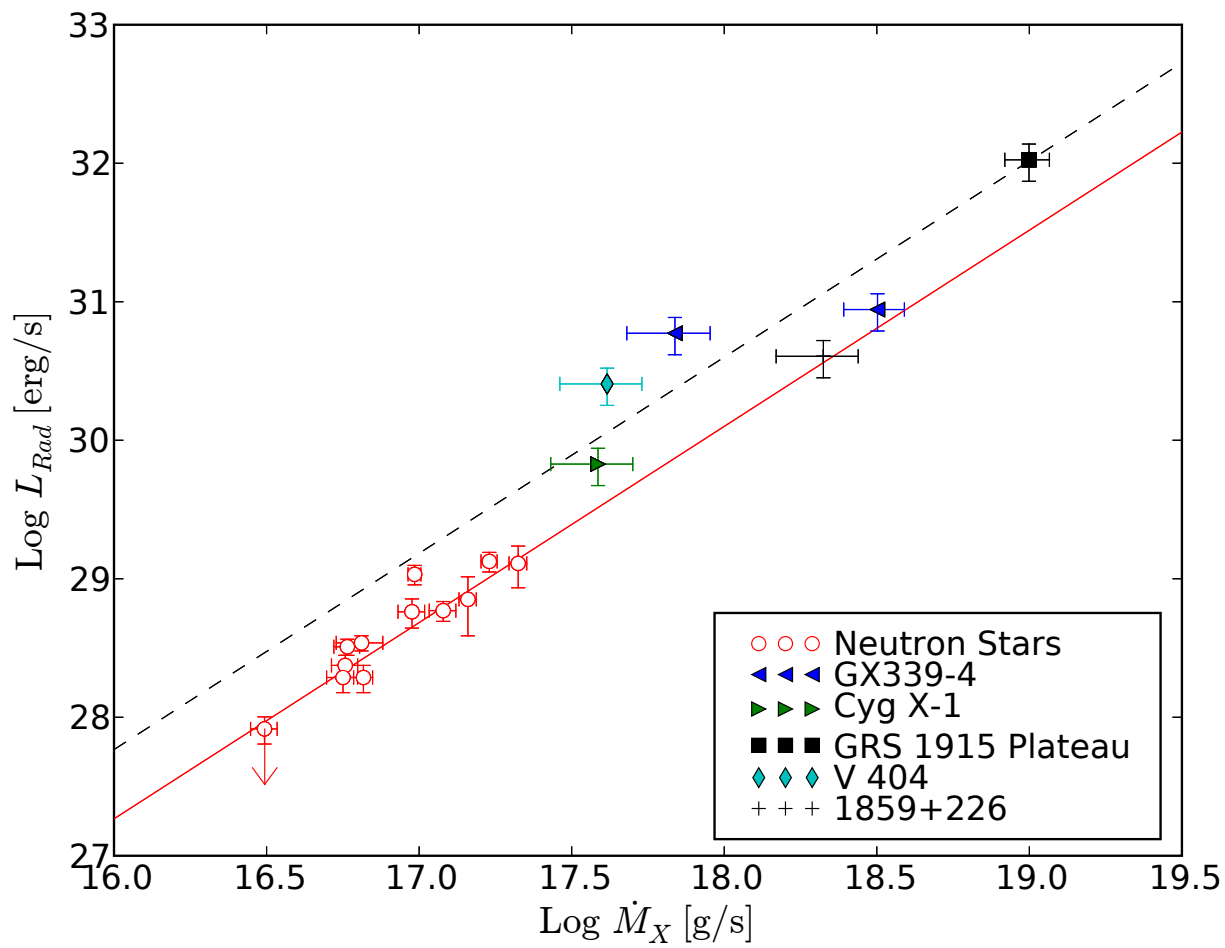

Figure 2: The correlation between accretion rate and radio luminosity for a sample of black hole and neutron star X-ray binaries. The lines indicate fits with gradient +1.4 , which is expected for the condition that jet power is linearly proportional to accretion rate. From Körding, Fender \& Migliari (2006).

Shortly afterwards two groups (Merloni, Heinz \& di Matteo 2003; Falcke, Körding \& Markoff 2004) independently established the existence of a plane linking $L_{\text {radio }}, L_{X}$ and mass $M$ of all accreting black holes, from X-ray binaries to AGN, and including Sgr A* (Fig 1). This should be considered one of the major steps in the unification of black hole accretion on all mass scales.

In the Merloni et al. formalism, the fundamental plane can be represented as:

$$
L_{\text {radio }} \propto L_{X}^{0.6} M^{0.8}
$$

where the power-law indices are fitted values to a large sample of XRBs and AGN.

The most recent refinements of the plane are presented in Gallo et al. (2006), Körding, Falcke $\&$ Corbel (2006). Criticisms of the plane have been rebuked by a consortium of all the original discovery authors, in Merloni et al. (2006).

\subsection{Calibrating the plane}

Of the three parameters of the fundamental plane, one is genuinely fundamental $(M)$, and one is a good indication of the total radiative output of the system and is therefore pretty fundamental $\left(L_{X}\right)$. However, the third parameter, $L_{\text {radio }}$ is merely a tiny tracer of the enormous power carried by the jets from these systems. The fact that it seems to correlate so perfectly with $L_{X}$ is itself quite amazing and indicates a remarkable stability and regularity in the jet formation process. 


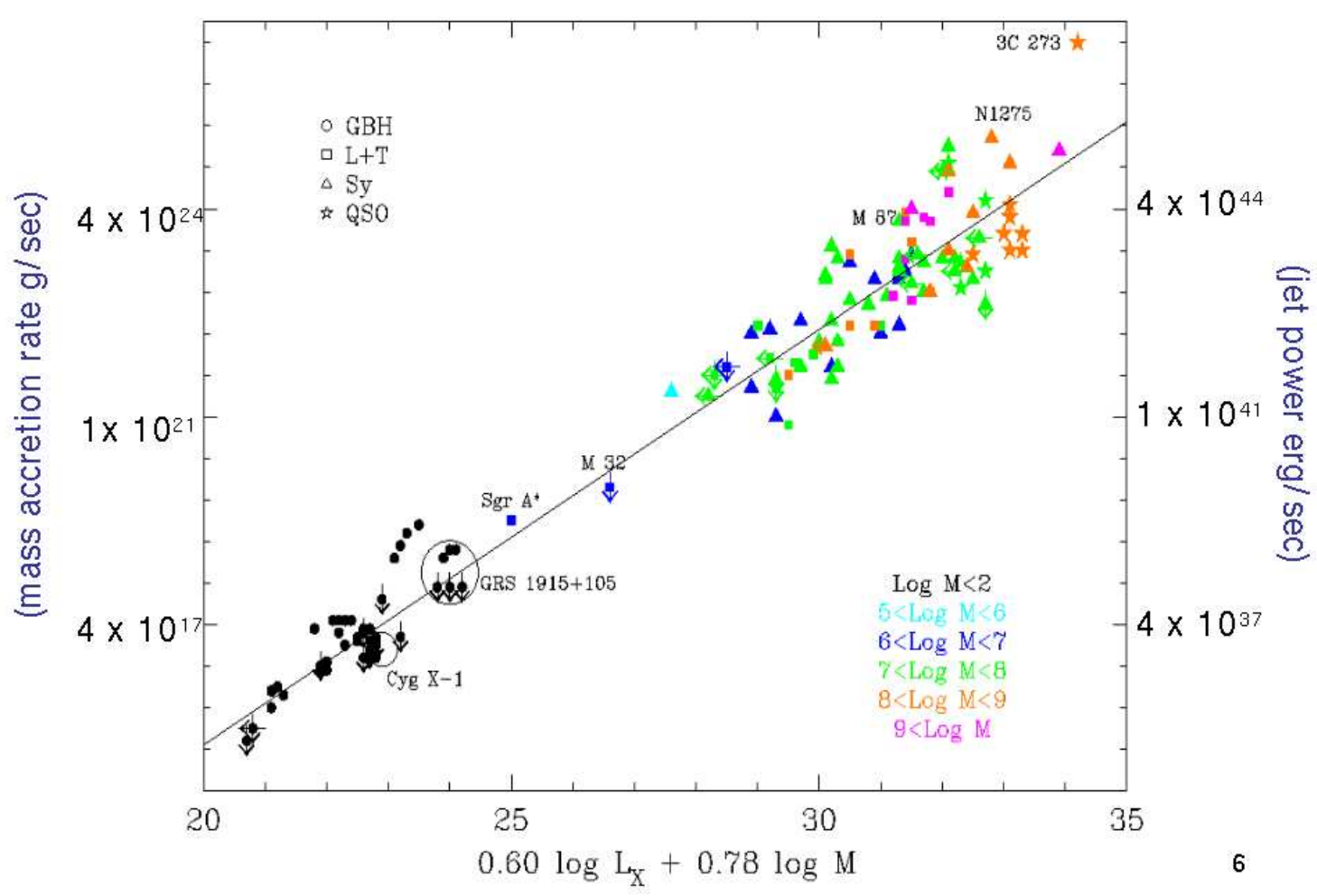

Figure 3: The 'calibrated' fundamental plane, where radio luminosity can be replaced by total jet power and/or mass accretion rate (see Fig 2), which are more physically useful numbers.

The plane would be a much better indicator of physical quantities and the flow of matter and power around the black hole if $L_{\text {radio }}$ could be replaced with, say, the total jet power $L_{J}$ or the mass accretion rate $\dot{m}$. In fact we can do both, based upon the relations established in Körding, Fender \& Migliari (2006; hereafter KFM03; see also e.g. Heinz \& Grimm 2005). In Fig 2 we present the relation between accretion rate (as determined from X-rays in radiatively efficient states) and radio luminosity from Körding, Fender \& Migliari (2006). In Fig 3 we use this and the related relation between jet power and radio luminosity from the same paper, to 'calibrate' the plane, by replacing the axes of Merloni et al. with the physical quantities of jet power (left) and accretion rate (right).

\subsection{What does the plane mean?}

The fundamental plane is an observational fact, a fit to measured quantities. But what does it mean ? In the following we outline what is probably the most straightforward interpretation.

If

$$
L_{\text {radio }} \propto \dot{m}^{1.4}
$$

which we consider to have been demonstrated in KFM03 (see our Fig 2), and

$$
L_{X} / L_{\mathrm{Edd}} \propto\left(\dot{m} / \dot{m}_{\mathrm{Edd}}\right)^{2}
$$




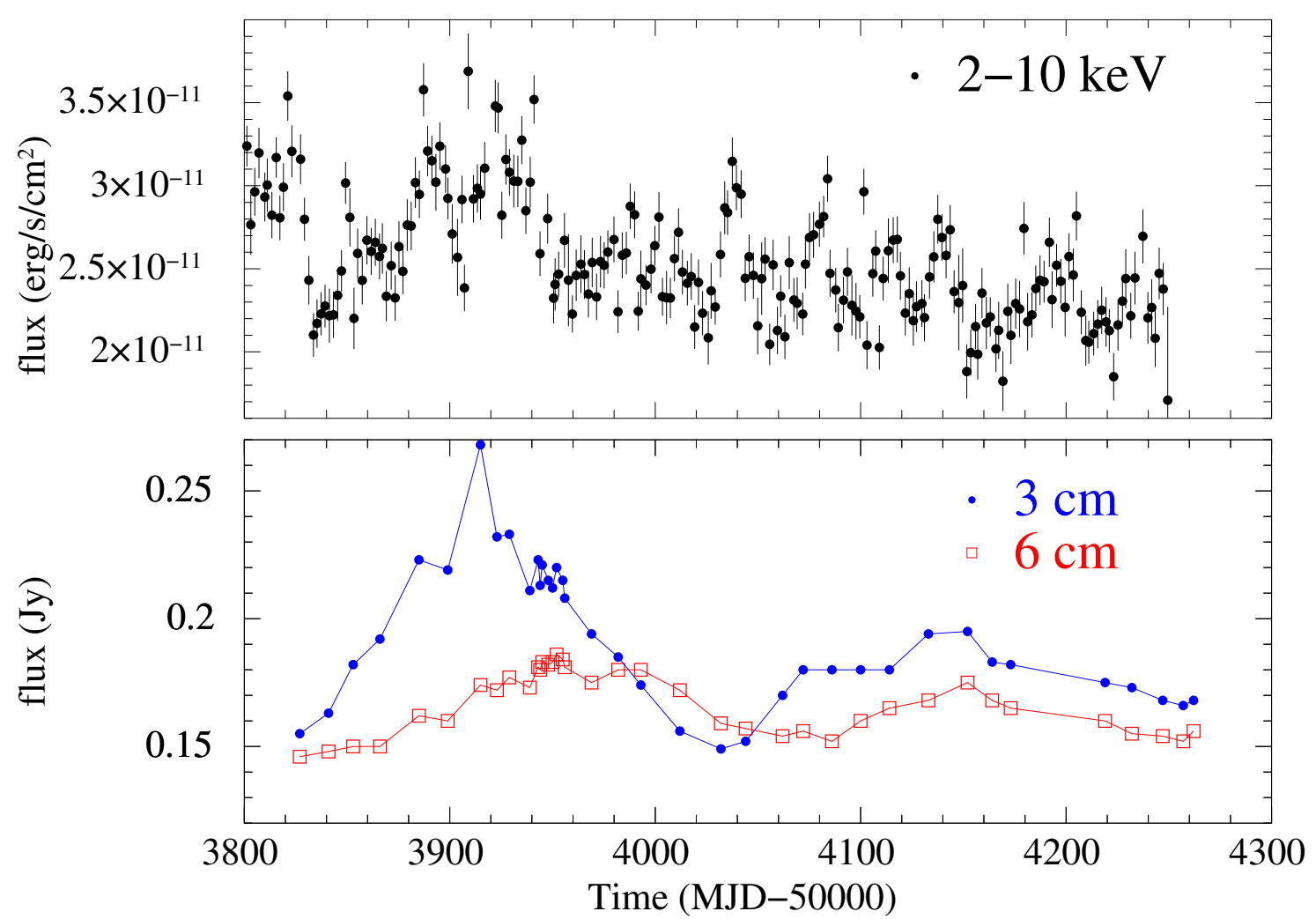

Figure 4: X-ray (RXTE) and radio (ATCA) monitoring of the low-luminosity AGN NGC 7213, for which monitoring continues. The goal of these observations is to look for lags and correlations between the two bands to try and recover the 'intrinsic' correlation which for AGN is so far only implied by the global effect (unlike for X-ray binaries, in which both are seen). Monitoring continues.

which is a general approximate solution for radiatively inefficient accretion (where the accretion flow knows which Eddington ratio it is at), then simple rearranging gives us:

$$
L_{\text {radio }} \propto L_{X}^{0.7} M^{0.7}
$$

which is within \pm 0.1 in power-law indices of the fitted plane.

This is not the only possible explanation for the existence of the fundamental plane, but it is probably the simplest. However, for it to work as an explanation, it requires that the fundamental plane be dominated by radiatively inefficient sources. Can that be right ? Körding, Fender \& Migliari (2006) argue that this is indeed the case (note that Merloni, Heinz \& di Matteo (2005) discuss this interpretation in a very similar way).

\section{Searching for the radio:X-ray correlation in a single AGN}

It is notable that in X-ray binaries both 'global' and 'intrinsic' correlations have been found, by which we mean that a range of points from a variety of sources traces out the same correlation as is observed from two individual sources (GX 339-4 and V404 Cyg). In the case of AGN, only the 'global' effect has been observed (although note the possible cyclic disc-jet coupling in 3C120 


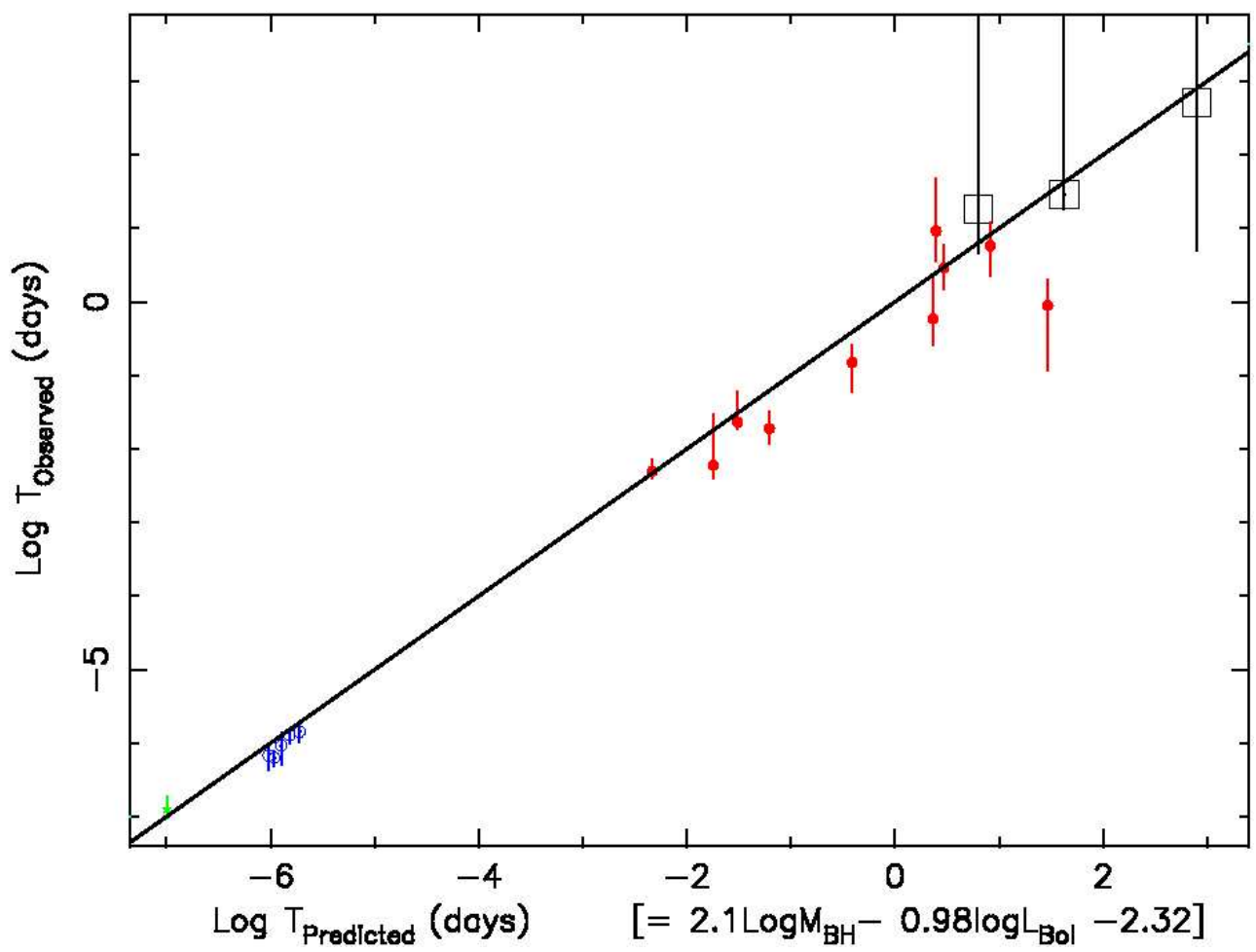

Figure 5: A second fundamental plane, essentially relating characteristic timescales to mass and accretion rate, from McHardy et al. (2006). More recently Körding et al. (2007) have shown this to extend 'hard state' black hole X-ray binaries and neutron star systems.

(Marscher et al. 2002)). Could it be possible to see something like the $L_{\text {radio }} \propto L_{\mathrm{X}}^{0.7}$ relation in a single AGN?

We have been attempting to do this with a program of X-ray (RXTE) and radio (ATCA) monitoring of the highly variable AGN NGC 7213 (see Fig 4). To date we have monitored the source for nearly a year and a half and see both the expected lags between the radio bands and some hints of correlation between radio and X-rays. Monitoring continues.

\section{Timing properties: another plane}

AGN X-ray timing studies started in the 1980s with the launch of EXOSAT, which probed AGN X-ray variability on time-scales up to a few days. It was soon established that on these time-scales AGN showed red-noise variability with steep unbroken PSDs (Lawrence et al. 1987, McHardy et al. 1987). However, McHardy (1988) noted using sparse long-term archival data that the PSDs appeared to flatten or break on longer time-scales, similar to the PSD shapes seen in BH XRBs. The launch of RXTE in 1995 allowed long-term light curves to be obtained with extremely good sampling and categorically proved the existence of PSD breaks on time-scales close to those expected by scaling the BH XRB break time-scales up by the AGN BH mass (e.g. Uttley et al. 2002, 2005; Markowitz et al. 2003, McHardy et al. 2004), although with some considerable scatter in the mass-time-scale relation. 
Recently, Migliari, Fender \& van der Klis (2005) established that for a small sample of X-ray binaries there was a positive correlation between radio luminosity and the frequencies of timing features. This was to be expected, since we are confident that on the whole both timing frequencies and radio luminosity are increasing functions of accretion rate (although we also know there are other, state-related, dependencies at high accretion rates).

We (McHardy et al. 2006) have now fitted a plane which relates mass and accretion rate to the break frequency in X-ray power spectra, such that

$$
T_{\text {break }} \propto M^{-2.1} L_{\mathrm{bol}}^{-1}
$$

Where $T_{\text {break }}$ is the break timescale, reciprocal of the break frequency, $M$ is black hole mass and $L_{\mathrm{bol}}$ is bolometric luminosity. All of the sources used in the correlation are believed to be in radiatively efficient states and so $L_{\mathrm{bol}}$ is used as a proxy for accretion rate. Using this substitution converted to accretion rate and integer power-law indices (i.e. $2 \sim 2.1$ ), we arrive at

$$
T_{\text {break }} \propto M /\left(\dot{m} / \dot{m}_{\text {Edd }}\right)
$$

revealing the expected linear correlation of break timescales with black hole mass, albeit for a fixed Eddington ratio of accretion rate. Our next goal is to see how well we can extend this relation to other accretion states and perhaps even other classes of object (Körding et al. 2007).

\section{Similarities in disc-jet coupling}

Black hole X-ray binaries seem to (nearly) always follow a pattern of behaviour in outburst similar to that sketched in Fender, Belloni \& Gallo (2004) and Homan \& Belloni (2005) (see Figs 6 and 7). However, it is clear that between different outbursts of the same source, or outbursts of different sources, the luminosities at which the hard $\rightarrow$ soft and soft $\rightarrow$ hard state transitions may occur can vary quite significantly (e.g. Belloni et al. 2006). As a result, an ensemble of X-ray binaries would present a pattern in the hardness:luminosity diagram which resembled a golf club ${ }^{1}$, with a long handle and a filled-in head. Such an ensemble is obviously what we're going to have to deal with if we want to be able to compare patterns of disc:jet coupling in XRBs with those in AGN.

In Körding, Jester \& Fender (2006) we have attempted to do this. First we constructed the Disc Fraction - Luminosity Diagram, in which hardness is replaced by the ratio of power law to total luminosity, a number which approaches zero for disc dominated soft states, and unity for hard states. This is necessary for a physical comparison, since the accretion discs temperature is a decreasing function of black hole mass, and for AGN does not contribute significantly in the X-ray band. We then simulated an ensemble of BHXRBs, based upon Fender, Belloni \& Gallo (2004) and the slight refinement (suggested in Belloni et al. 2006) that the 'jet line' might be diagonal in such a diagram. This was then compared to a sample of AGN from the SDSS DR5 for which there were X-ray detections and either radio detections or limits, plus a sample of low-luminosity AGN (LLAGN). The similarity was striking (see Fig 6) and suggests that the radio loudness is

\footnotetext{
${ }^{1}$ or a turtle's head, as was suggested in a bar at the Texas Symposium in 2004. .
} 

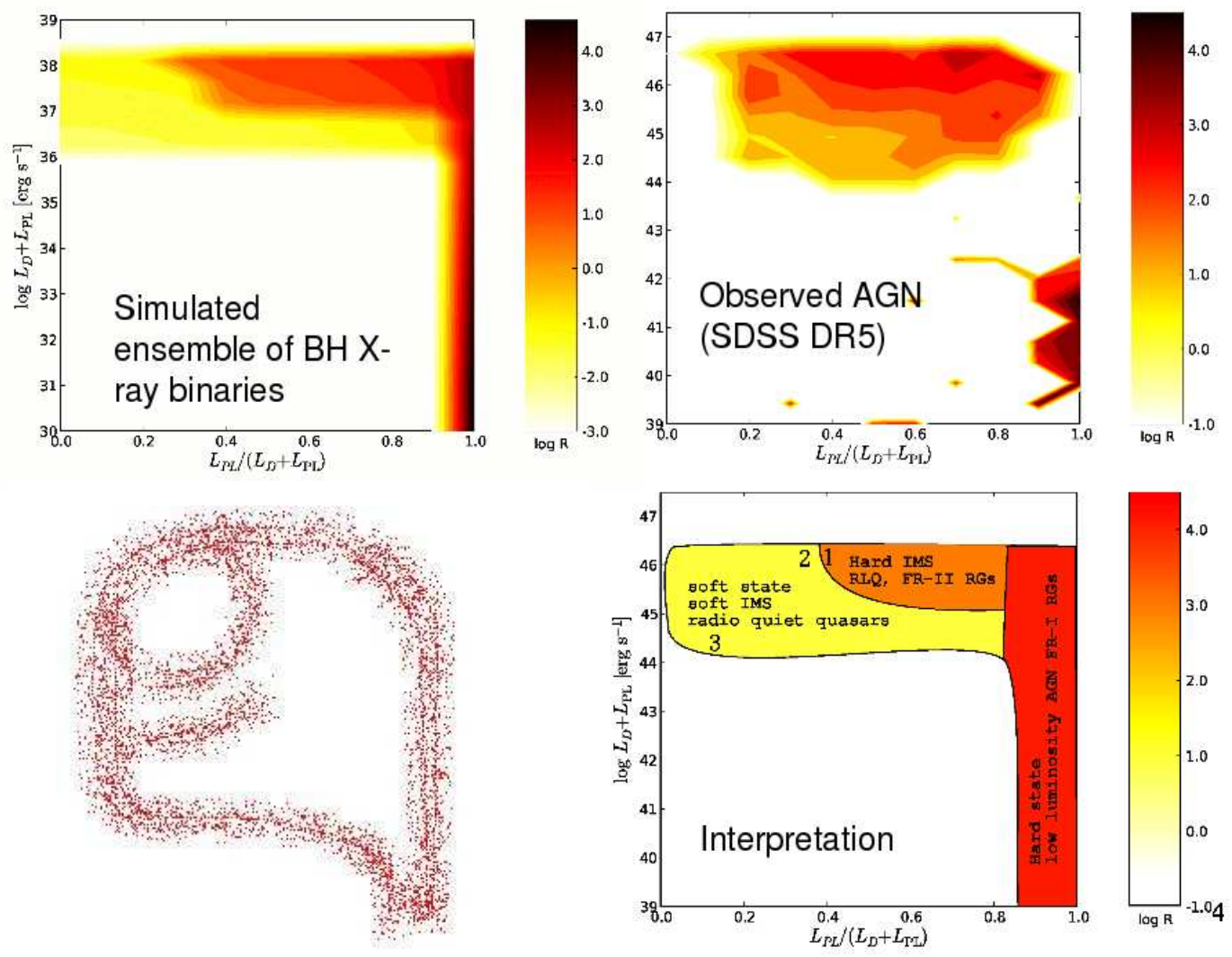

Figure 6: A comparison of the disc:jet coupling in X-ray binaries with that in AGN, based upon the Disc Fraction Luminosity Diagram (DFLD; see Körding, Jester \& Fender 2006). Based upon the disc:jet model for black hole X-ray binaries presented in Fender, Belloni \& Gallo (2004), an example track for which is shown in the lower left panel, a simulated ensemble of X-ray binaries was produced (upper left panel). This was compared with a sample of SDSS quasars and low luminosityt AGN (upper right panel) and a striking similarity revealed. The lower right panel offers an explanation for the similarities between the different classes of object.

determined by the combination of 'state' and luminosity in a similar way for accreting black holes of all masses. Note that, while it is tempting to consider, the diagram does not indicate that AGN necessarily follow the same anti-clockwise loop in the diagram as XRBs: the motion in such loops could possibly be dominated by disc instabilities which may not apply to AGN. What it does indicate is that when an AGN finds itself in a particular accretion 'state', whether disc or corona dominated or some mix of the two, the jet it produces will be comparable to that which a XRB would make in the same state.

\section{Beware of cheap imitations (or: Neutron stars can do it too)}

It has already been known for some years that neutron star X-ray binaries (NSXRBs) are also radio sources. Migliari \& Fender (2006) present a review of such observations, including cases where the radio emission has been resolved into jets, and compare them with those from black 

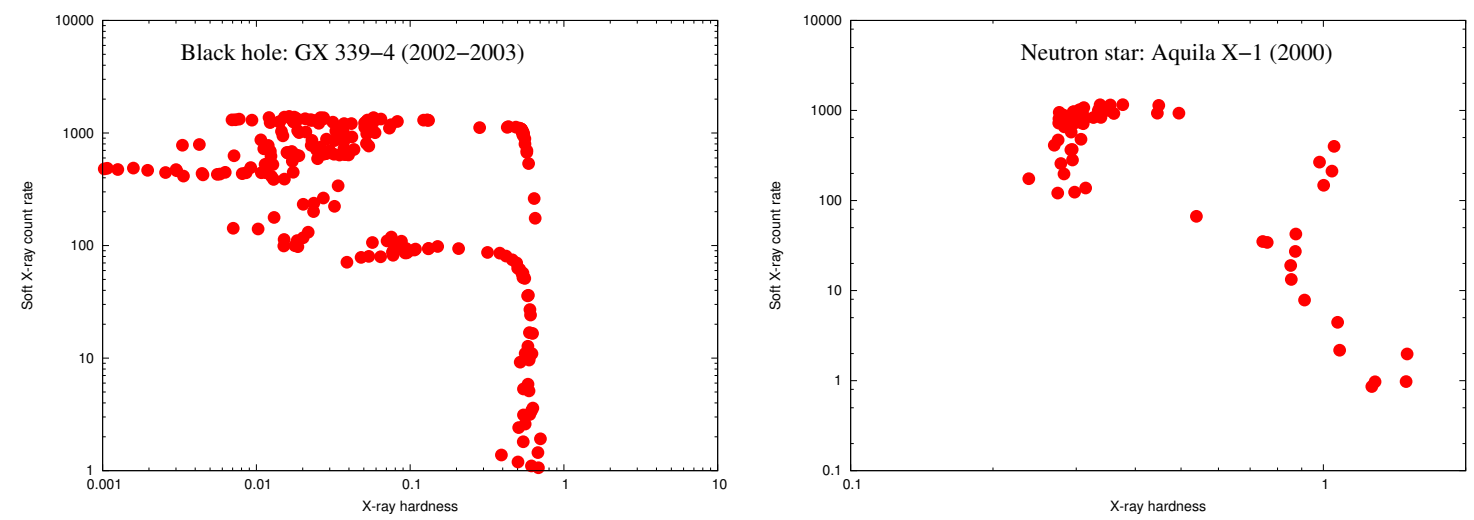

Figure 7: $\mathrm{X}$-ray hardness intensity diagrams for the two $\mathrm{X}$-ray binaries in outburst. The left panel is the 2002-2003 outburst of the black hole X-ray binary GX 339-4 (Homan \& Belloni 2005). The right panel is the 2000 outburst of the neutron star X-ray binary Aquila X-1 (Maitra \& Bailyn 2003). The similarity in the pattern of outburst, notably the hysteretical state changes, is clear. Note that the X-ray colours are defined slightly differently for the two sources.

hole X-ray binaries. It is clear that at least at high accretion rates, NSXRBs can also produce relativistic jets which would not look out of place in a black hole system. Furthermore, as already noted, Körding, Fender \& Migliari (2006) argue that, within a factor of a few, NSXRB jets are as powerful as those from BHXRBs for a given accretion rate (in hard states).

What about the patterns of outbursts - perhaps those could be used as a discriminant? It seems not: Maitra \& Bailyn (2003) present the HID for an outburst of the neutron star X-ray transient Aql $\mathrm{X}-1$. The HID, when plotted on similar axes to those of the black holes (Fig 7) is striking similar (see also van der Klis 2006). This indicates that the pattern of the outburst is not dominated by any properties specific to black holes such as a static limit or event horizon, but rather by properties common to both classes of object, such as disc instabilities and accretion into a deep gravitational potential. So while there are some patterns of behaviour - e.g. the Z sources - which seem to be unique to NSXRBs, overall it does not seem that we can attribute any key property of relativistic jet formation, or its coupling to accretion, as arising due to some property unique to black holes.

\section{Conclusions}

The scaling of black hole accretion with mass, from X-ray binaries to Quasars, has evolved from a clear concept and idea in the 1970s, to a something with a sound observational footing in the 1990s, and eventually to a field with measured quantitative scalings with predictive power in the 2000s. So what do we do with these scaling relations?

The stated goal, beyond testing the hypothetical simplicity of black hole accretion, has often been that we can use the relatively rapid temporal evolution of black hole X-ray binaries to understand something about the life cycles of AGN. Happily the importance of this potential insight has only grown in recent years with the growing acceptance that feedback from AGN directly shapes the evolution of the host galaxy (e.g Best et al. 2005 and references therein; see also Silk \& Rees 1998) and in some cases, clusters in which the AGN are embedded. There are already some examples of linkage between the fields. Based on knowledge of the relation of jet production and 
mass accretion rate (caveat state hysteresis at high accretion rates), one can for example take AGN luminosity functions and use them to estimate the total amount of kinetic feedback associated with the accretion process (see Merloni \& Heinz (2007) and Körding, Jester \& Fender (2007)).

Finally it is of interest to note that as the Universe continues to evolve, we will move towards a time in which the release of gravitational energy is dominated by jets, rather than radiation, as black holes accrete at lower and lower mean Eddington ratios. The future is not bright.

\section{Acknowledgments}

RPF would like to acknowledge useful conversation with Annalisa Celotti, Heino Falcke, Elena Gallo, Jeroen Homan, Sebastian Jester, Christian Kaiser, Tom Maccarone, Sera Markoff, Dave Meier, Simone Migliari and many others both at the Como workshop and further back in the mists of time.

\section{References}

[1] Belloni T., et al., 2006, 367, 1113

[2] Best P.N., Kauffmann G., Heckman T. M., Brinchmann J., Charlot S., Ivezic Z.; White S. D. M., 2005, MNRAS, 362, 25

[3] Brocksopp C., Corbel S., Fender R., Rupen M., Sault R., Tingay S., Hannikainen D., O'Brien K., $2005,356,125$

[4] Carter B., 1973, Properties of the Kerr metric, in DeWitt C. and DeWitt B.S., 'Black Holes', Proceedings of the 1972 session of Ecole d'ete de physique theorique, Gordon \& Breach, New York

[5] Corbel S., Fender R.P., Tzioumis A.K., Nowak M., McIntyre V., Durouchoux P., Sood R., 2000, A\&A, 359, 251

[6] Corbel S., Nowak M.A., Fender R.P., Tzioumis A.K., Markoff S., 2003, A\&A, 400, 1007

[7] Falcke H., Biermann P.L., 1996, A\&A, 308, 321

[8] Falcke H., Körding E., Markoff S., 2004, A\&A, 414, 895

[9] Fender R., Belloni T., Gallo E., 2004, MNRAS, 355, 1105

[10] Gallo E., Fender R.P., Pooley G.G., 2003, MNRAS, 344, 60

[11] Gallo E., Fender R.P., Miller-Jones J.C.A., Merloni A., Jonker P., Heinz S., Maccarone T., van der Klis M., 2006, 370, 1351

[12] Heinz S., Grimm S.J., 2005, ApJ, 633, 384

[13] Heinz S., Sunyaev R., 2003, MNRAS, 343, L59

[14] Homan J., Belloni T., 2005, Ap\&SS, 300, 107

[15] Körding, Falcke H., Corbel S., A\&A, 456, 439

[16] Körding, Fender R., Migliari S., 2006, MNRAS, 369, 1451

[17] Körding, Jester S., Fender R., 2006, MNRAS, 372, 1366

[18] Körding, Jester S., Fender R., 2007, MNRAS submitted 
[19] Körding E.G., Migliari S., Fender R., Belloni T., Knigge C., McHardy I., 2007, MNRAS, in press

[20] Lawrence, A., Watson, M. G., Pounds, K. A., Elvis, M., 1987, Nature, 325, 694

[21] McHardy I., Körding E., Uttley P., Knigge C., Fender R., 2006, Nature, 444, 730

[22] McHardy, I. M., Czerny, B., 1987, Nature, 325, 696

[23] McHardy, I. M., 1988, Mem. Soc. Astron. Ital., 59, 239

[24] McHardy, I. M., Papadakis, I. E., Uttley, P., Page, M. J., Mason, K. O., 2004, MNRAS, 348, 783

[25] Maccarone T.J., Fender R.P., Ho L.C., 2005, From X-ray binaries to Quasars: Black Holes on All Mass Scales, Reprinted from Ap\&SS volume 300, Nos 1-3, Springer

[26] Markoff S., Falcke H., Fender R., 2001, A\&A, 372, L25

[27] Markoff S., Nowak M., Corbel S., Fender R., Falcke H., 2003, A\&A, 397, 645

[28] Marscher A.P., Jorstad S.G., Gomez J.-L., Aller M.F., Terasranta H., Lister M.L., Stirling A.M., 2002, Nature, 317,625

[29] Markowitz, A., et al., 2003, ApJ, 593, 96

[30] Maitra D., Bailyn C.D., 2004, ApJ, 608, 444

[31] Merloni A., Heinz S., 2007, Black Holes from Stars to Galaxies - Across the Range of Masses. Edited by V. Karas and G. Matt. Proceedings of IAU Symposium 238, held 21-25 August, 2006 in Prague, Czech Republic. Cambridge, UK: Cambridge University Press, pp.65-70

[32] Merloni A., Heinz S., di Matteo T., 2003, MNRAS, 345, 1057

[33] Merloni A., Heinz S., di Matteo T., 2005, Ap\&SS, 300, 45

[34] Merloni A., Körding E., Heinz S., Markoff S., di Matteo T., Falcke H., 2006, New Astronomy, 11, 567

[35] Migliari S., Fender R., 2006, MNRAS, 366, 79

[36] Migliari S., Fender R., van der Klis M., 2005, 363, 112

[37] Mirabel I.F., Rodriguez L.F., 1994, Nature, 371, 46

[38] Mirabel I.F., Rodriguez L.F., 1999, ARA\&A, 37, 409

[39] Mirabel I.F., Rodriguez L.F., Cordier B., Paul J., Lebrun F., 1992, Nature, 358, 215

[40] Pounds K.A., Done C., Osborne J.P., 1995, MNRAS, 277, L5

[41] Sams B.J., Eckart A., Sunyaev R., 1996, Nature, 382, 47

[42] Shakura N.I., Sunyaev R.A., 1976, MNRAS, 175, 613

[43] Silk J., Rees M., 1998, A\&A, 331, L1

[44] Uttley, P., McHardy, I. M., \& Papadakis, I. E., 2002, MNRAS, 332, 231

[45] Uttley, P. McHardy, I. M., 2005, MNRAS, 363, 586

[46] van der Klis M., 2006, Compact Stellar X-ray Sources, Cambridge Astrophysics Series 39, Cambridge University Press, pp.39-98 